\title{
El Rorschach en el siglo XXI: La evaluación de la representación mental
}

Sydney J. Blatt

Universidad de Yale

El test de Rorschach tiene un potencial considerable para contribuir al estudio de procesos cognitivos, especialmente el mundo representacional y de cómo los individuos construyen el significado. La representación del yo y del otro es actualmente uno de los principales temas en la investigación del apego y de la cognición social, y es muy relevante para la psicología clínica y la psiquiatría clínica porque provee una forma de comprender la psicopatología que va más allá del simple listado de sintomas manifiestos que es tan predominante en la psiquiatría descriptiva hoy en día. El nuevo énfasis en la representación mental debe permitirnos ampliar nuestro conocimiento de la psicopatología y descubrir nuevas formas para evaluar sistemáticamente a un amplio espectro de los trastornos psicológicos.

Test de Rorschach / métodos proyectivos / evaluación de la personalidad / procesos cognitivos

\section{The Rorschach in the 21st century: The assessment of mental representation}

The Rorschach test has considerable potential to contribute to the study of cognitive processes, especially the representational world and of how individuals construct meaning. The representation of self and other is currently a major topic in attachment research and in social cognition and is very relevant for clinical psychology and psychiatry because it provides a way of understanding psychopathology that goes beyond the simple listing of manifest symptoms that is so prevalent today in descriptive psychiatry. This new emphasis on mental representation should enable us to extend our understanding of psychopathology and to discover new ways of systematically assessing a wide range of psychological disturbances.

Rorschach test / projective methods / personality evaluation / cognitive processes

Traducción: Javier Díaz-Albertini Figueras. 
Herman Rorschach desarrolló sus ideas acerca de la evaluación de la personalidad en la primera mitad del siglo XX, cuando la psicología -como disciplina académica- estaba primordialmente preocupada por la sensación, la percepción, la predicción y el control de la conducta manifiesta. Los procesos perceptivos de la visión y audición eran los focos principales de la investigación psicológica. Los procesos cognitivos y las operaciones de la mente eran vistos como experiencias subjetivas que ocurrían al interior de lo que se denominaba -en aquellos tiempos- una "caja negra" impenetrable. Como estos procesos no eran observables, se les consideraba como inapropiados para el estudio científico. La psicología se definía a sí misma como una ciencia de la conducta y la investigación psicológica enfocaba los procesos perceptivos y la investigación de la unidad estímulo-respuesta, del arco reflexivo, con poca atención prestada a los procesos que mediaban la relación entre la entrada del estímulo y la respuesta conductual. De ahí que no sea sorprendente que en 1921, cuando Hermann Rorschach descubrió el valor de manchas de tinta ambiguas para la evaluación de la personalidad, considerara su procedimiento como una "prueba perceptiva" y trató de ligar la tendencia del uso de diferentes aspectos del campo perceptivo -la forma, el color y la sombra de las manchas de tinta- con ciertos tipos de respuestas conductuales. En los años sucesivos, se ha acumulado un cuerpo de conocimiento que apoya a muchas de las presunciones básicas de Rorschach acerca del vínculo entre cier- tas proclividades perceptivas y un rango amplio de respuestas conductuales.

El valor de la contribución de Rorschach, sin embargo, no estriba en la formulación del vínculo entre tendencias perceptivas y respuesta conductual, sino en el desarrollo de un procedimiento que confronta al individuo con estímulos ambiguos en circunstancias inciertas y que nos permite observar sistemáticamente cómo las personas organizan la realidad y construyen significado en situaciones relativamente desestructuradas. Al respecto, el procedimiento de Rorschach es congruente con el énfasis contemporáneo puesto en procesos cognitivos y construccionismo, y, por ende, el Rorschach tiene un potencial considerable para contribuir al estudio de temas que se han convertido en el foco central de mucha de la ciencia psicológica en la segunda mitad del siglo XX.

En esta época, la psicología se convirtió en algo más que una ciencia conductual -se convirtió en una ciencia de la mente-, la investigación de cómo las personas construyen significado $y$ cómo estos sistemas de significados se expresan en conductas. Influenciados por el altamente aclamado texto de Charles Osgood de 1953, Method and theory in experimental psychology, los psicólogos a mediados de siglo empezaron a considerar los procesos que podrían estar ocurriendo en la "caja oscura" -los "procesos mediacionales (Osgood, 1953) que establecían los vínculos entre el estímulo y la respuesta conductual-. A principios de los años sesenta, la psicología comenzó lo que 
ha sido denominado como la "revolución cognitiva" (Gardner, 1985) y muchas de las subáreas de la psicología contemporánea (por ejemplo: psicología del desarrollo, psicología clínica) comenzaron a interesarse en estructuras cognitivas -esquemas, guiones, planes, plantillas, modelos de trabajo interno y representaciones mentales-, especialmente en cómo estas estructuras cognitivas son establecidas, cómo influyen en el desarrollo psicológico y cómo son expresadas en relaciones interpersonales.

Estas estructuras cognitivas se han convertido en constructos teóricos centrales no sólo para la ciencia cognitiva y la psicología del desarrollo y social, sino también en la teoría y práctica psicoanalíticas. Investigaciones en psicoanálisis y en psicología del desarrollo cognitivo indican que los niños, sobre la base de interacciones tempranas con sus cuidadores primarios, construyen esquemas cognitivo-afectivos del self y el otro y que estas representaciones mentales se desarrollan durante el ciclo vital. Estos esquemas tienen componentes cognitivos, afectivos y experienciales conscientes e inconscientes que se derivan de experiencias interpersonales tempranas y significativas. Los esquemas cognitivo-afectivos también reflejan el nivel de desarrollo del individuo y los aspectos importantes de la vida psíquica, como los impulsos, afectos, impulsos y fantasías. Estos esquemas cognitivo-afectivos pueden implicar representaciones verídicas de realidad consensual, construcciones idiosincrásicas y únicas, o dis- torsiones primitivas o patológicas que sugieren psicopatología (Blatt, 1991, 1995). Ambas teorías, la psicoanalítica y la del desarrollo con enfoque cognitivo, intentan explicar las formas como los individuos establecen, mantienen y revisan estos sistemas de significados (esquemas, planes, libretos o representaciones). Las formulaciones y hallazgos de la teoría psicoanálitica relaciones objetales y de la psicología del desarrollo son consistentes con el énfasis reciente de la ciencia cognitiva, del procesamiento de información, y de la cognición social, de que estos esquemas son prototipos heurísticos que proveen las bases para la interacción social (Blatt, Auerbach y Levy, 1997).

Los esquemas del self y el otro son construidos en transacciones interpersonales que comienzan en la relación del infante con quien asume su cuidado, y estos esquemas normalmente se desenvuelven como parte de un proceso natural de maduración en respuesta a demandas y perturbaciones del entorno. Cuando las demandas del entorno son apropiadas para la edad y no muy severas, las estructuras cognitivas existentes evolucionan para acomodar las perturbaciones experimentadas. Estas acomodaciones resultan en la construcción de estructuras cognitivo-afectivas más maduras que usualmente se desenvuelven en una secuencia de desarrollo bien definida: de representadas, afectivas y físicas a simbólicas y abstractas. Los esquemas crecientemente maduros efectivamente organizan, moldean y guían la subsiguiente conducta interpersonal. Pero perturbaciones severas o 
inapropiadas del desarrollo pueden desbordar las capacidades del niño para acomodarse y comprometer el desarrollo de estas capacidades de representación. Conmociones o deterioros específicos a la estructura y contenido de estos esquemas cognitivo-afectivos son temas centrales en diversas formas de psicopatologías (Blatt, 1991, 1995).

\section{REPRESENTACIONES MENTALES EN LA}

\section{TEORÍA DE APEGO Y LA TEORÍA PSICOANALÍTICA OBJETO RELACIONAL}

Las teorías e investigaciones cognitivas del desarrollo y psicoanalítica demuestran que la estructura y el contenido de los esquemas cognitivo-afectivos se derivan de las sutilezas de la afinación relacional entre el cuidador y el infante, expresados en patrones de enganche y desenganche en los primeros meses de la vida (Beebe, 1986; Beebe \& Lachmann, 1988; Stern, 1985) y en los patrones de apego y separación en la primera mitad del segundo año (Ainsworth, 1982; Bowlby, 1988). Las experiencias de crianza relativamente satisfactorias facilitan el desarrollo de un sentido diferenciado y cohesivo del self y los otros, que provee las bases para el desarrollo de la capacidad para entablar relaciones interpersonales crecientemente maduras. La investigación en patrones de apego en los dos primeros años de vida sugieren que el niño establece modelos internos de trabajo (IWNs; véase Bowlby, 1969, 1988; Main, Kaplan, \& Cassidy, 1985), de relaciones de apego que son relativa- mente estables en el tiempo (Ainsworth, 1982; Bretherton, 1985) e influyen sobre un rango amplio de conductas, aun entrada en la adultez.

Estos modelos han sido definidos como

... un conjunto de reglas conscientes e inconscientes para la organización de información relevante para (...) experiencias, sentimientos e ideaciones relacionadas al apego (Main et al., 1985, p. 67).

Éstos funcionan como plantillas "sorprendentemente poderosas" que están "relacionados no sólo a patrones individuales en la conducta no verbal, sino también a patrones del lenguaje y estructuras mentales" (Main et al., 1985, p. 67). Los modelos internos de trabajo formados tempranamente en la vida varían en su flexibilidad, adaptabilidad y madurez, pero son centrales en el desarrollo de un sentido del self y los otros e influyen profundamente en la naturaleza y calidad de las relaciones interpersonales durante la vida. Estos esquemas son guías heurísticas que organizan experiencias, modulan el afecto y proveen dirección para la conducta subsecuente. Se convierten en estructuras psicológicas perdurables o plantillas que procesan y organizan información y promueven la asimilación de nuevas experiencias en las estructuras mentales existentes (Blatt \& Lerner, 1983a, 1983b). El desarrollo psicológico puede ser visto, entonces, como el desenvolvimiento epigenético progresivo de nuevos esquemas cognitivos que evolucionan de estructuras cognitivas más tempranas. 


\section{Cuadro $\mathrm{N}^{\circ} 1$ \\ Niveles de esquemas cognitivos*}

\section{Nivel de desarrollo}

\section{Indicador conductal}

I. Constancia de límites

II. Constancia (6 a 8 meses) de reconocimiento (libidinal)

III. Constancia de evocación (16-18 meses)

IV. Constancia de self y objeto (30 a 36 meses)

V. Pensamiento concreto operacional (5 años)

VI. Pensamiento formal operacional (11-12 años)

VII. Auto identidad (adolescencia tardía, adultez temprana)

VIII. Integridad
Respuesta sonriente, inicia compromiso con otros.

Ansiedad al extraño, diferenciación entre personas, apego libidinal a un individuo específico.

El sentido del objeto no se encuentra inmediatamente presente en el campo perceptivo. Anticipación del desplazamiento invisible (Piaget), se inicia la separación del agente cuidador (Mahler).

Conceptos estables del self y el otro, expresado en el uso de términos como "mío", "me" y "yo".

Capacidad para la coordinación de varias dimensiones simultáneamente. Capacidad para la anticipación, transformación, conservación y reversibilidad de aspectos externos manifiestos. Configuraciones triádicas y el surgimiento de un concepto de "nosotros".

Transformación, reversibilidad y conservación de aspectos internos abstractos, dimensiones y procesos tales como valores y principios. Reconocimiento de que uno construye significado y sentido de la realidad. Apreciación del relativismo personal y cultural.

Síntesis e integración de expresiones maduras tanto de la individualidad como del relacionamiento en una capacidad de ser íntimo con otro y a contribuir a un colectivo sin perder su propia individualidad.

Surgimiento de un sentido más completo del "nosotros" (eso es, "el self en relación" [Chordorow, 1978, 1989; (adultez madura) Gilligan, 1982; Miller, 1976] o "individualismo ensamblado" [Sampson, 1985, 1988]).

* Tomado de Blatt, S.J. (1995). Representational structures in psychopathology, en Cicchetti, D. y S. Toth, Rochester (eds). Rochester Symposium on Developmental Psychopathology. Emotion, cognition, and representation. Vol. 6. New York: University of Rochester Press, pp. 1-33. 
Los estudios empíricos sobre las cualidades de los modelos en niños, adolescentes y adultos se asemejan a las formulaciones de teóricos psicoanalíticos objeto-relacionales que consideran a las representaciones cognitivo-afectivas del self y los otros como estructuras psicológicas fundamentales en el desarrollo y organización de la personalidad (Beres \& Joseph, 1970; Blatt, 1974; Blum, 1961; Horowitz, 1972; Jacobson, 1964; Sandler \& Rosenblatt, 1962). La comprensión de estas representaciones del self y los otros en la teoría objetorelacional se sustenta primordialmente en experiencias clínicas con adultos, mientras que la formulación de modelos internos de trabajo de la teoría de apego se deriva sobre todo del estudio de niños y adultos normales. Una integración de las teorías objeto-relacional y de apego, sin embargo, ofrece la posibilidad de especificar las relaciones complejas entre las dimensiones interpersonales, afectivas y cognitivas en el desarrollo psicológico. Por ejemplo, una mayor complejidad en las representaciones permite una mejor regulación de afectos, un mayor nivel de integración y una mayor tolerancia de la ambivalencia hacia otros (Gruen \& Blatt, 1990; Diamond, Kaslow, Coonerty \& Blatt, 1990; Levy, Blatt \& Shaver, 1998).

La teoría de desarrollo cognitivo (por ejemplo, Piaget y Werner) y la teoría psicoanalítica objeto relacional (por ejemplo, Fraiberg, A. Freud, Jacobson y Mahler) pueden utilizarse para identificar varios puntos nodales en el desarrollo estructural de las representacio- nes mentales. Estos puntos nodales son los siguientes:

- Constancia de límites, o sea la capacidad para establecer y mantener un sentido de separación entre el self y el otro, entre el self y el no self.

- Constancia de reconocimiento o emocional, en la cual uno es capaz de establecer y mantener un apego emocional consistente con una persona particular.

- Constancia evocativa o de objeto, en la cual uno es capaz de establecer y mantener una relación emocional positiva con un otro significativo, aún cuando el otro no se encuentra en el campo perceptivo inmediato o cuando uno está en conflicto con esa persona.

- Constancia del self, en la cual uno tiene una representación consolidada, cohesiva y estable de sí mismo como diferente y distinguible de los demás y como perdurable en el tiempo y espacio, a pesar de sus estados emocionales.

- Pensamiento operativo, en el cual uno es capaz de coordinar relaciones entre varias dimensiones y, por ende, es capaz de considerarse a sí mismo al interior de la configuración interpersonal triade (por ejemplo, en su familia, y ulteriormente en contextos sociales más amplios). Con el pensamiento operativo, el sentido del self y el sentido del otro pueden extenderse a un sentido de "nosotros" (Blatt, 1995). 


\section{IMPLICANCIAS PARA LA PSICOPATOLOGÍA}

Varias formas de psicopatología, desde la esquizofrenia hasta las neurosis, comprometen deterioros diferenciales en la estructura de representaciones mentales, eso es, en el desarrollo de esquemas cognitivo-afectivos del self y el otro. Extensivos hallazgos de la investigación y de informes clínicos, muestran que un rango amplio de síntomas y muchos de los disturbios cognitivos, preceptuales e interpersonales observados con frecuencia en la esquizofrenia, pueden ser entendidos como disrupciones en la capacidad de establecer y mantener límites (Blatt \& Ritzler, 1974; Blatt \& Wild, 1976), y de una inhabilidad de establecer y mantener constancia emocional (o de reconocimiento) en sus relaciones interpersonales (Blatt, Schimek \& Brenneis, 1980). Las respuestas de contaminación en el Rorschach son un ejemplo primario del trastorno de límites en la esquizofrenia. En la respuesta de con- taminación, dos conceptos o preceptos independientes se fusionan en un todo idiosincrásico (por ejemplo, la respuesta de "una mano de conejo" porque se parece a un conejo y se parece a una mano en el detalle inferior de la lámina).

En contraste, muchos de los síntomas y deterioros en la patología borderline pueden entenderse como trastornos en la capacidad de establecer y mantener constancia de objeto y self (o evocar o recordar) (Adler \& Bluie, 1979; Auerbach \& Blatt, 1996). La respuesta de la confabulación, la forma más típica de desorden en el pensamiento en el Rorschach para desórdenes fronterizos (borderline) de la personalidad (Blatt \& Auerbach, 1988; Blatt \& Schichman, 1983; Lerner, Sugarman \& Barbour, 1985; Wilson, 1985), es un ejemplo fundamental del impacto de trastornos en la constancia evocativa en el funcionamiento psicológico de pacientes borderline. En la respuesta de la confabulación, las asociaciones idiosincrásicas

\section{Cuadro $\mathrm{N}^{\circ} 2$}

Psicopatología y deterioro en representaciones mentales

\begin{tabular}{ll}
\hline Psicopatología & Representaciones \\
\hline Esquizofrenia & Deterioro en la constancia de límites y \\
& reconocimiento (emocional). \\
Desorden de personalidad fronterizo & Deterioro en la constancia de evocación (objeto y self). \\
Neurosis y depresión & Deterioro en el pensamiento operacional (deterioro en el \\
& sentido de "nosotros").
\end{tabular}


y reacciones afectivas intensas abruman el pensamiento y la percepción realista, de tal manera que una inicial percepción precisa o respuesta realista es inapropiadamente inculcada con afecto intenso o excesiva elaboración (por ejemplo, "alguien inclinándose y gritando en agonía, duele sólo mirarlo" o "una mujer cercenada por osos, la están rasgando en pedazos"). El pensamiento exagerado y vistoso expresado en las respuestas de confabulación de los pacientes fronterizos reflejan el intento de estos pacientes de compensar su déficit en constancia evocativa (Adler \& Buie, 1979; Blatt \& Shichman, 1983). Las imágenes vívidas y exageradas y los conceptos exageradamente simbólicos y polarizados (por ejemplo, la idealización extrema y la denigración grotesca) son algunas de las formas mediante las cuales el paciente fronterizo hace que las cosas sean demasiado dramáticas y vívidas como intento de mantener un sentido estable de los otros y un sentido cohesivo, aunque frecuentemente negativo, de sí mismo. A pesar de la ideación y el afecto doloroso frecuentemente reflejados en las respuestas de confabulación, estas imágenes por lo menos proveen algún grado de estabilidad y un sentido tentativo de continuidad y constancia en un mundo psicológico inestable. Las investigaciones empíricas del desorden en el pensamiento de pacientes seriamente trastornados (por ejemplo, Lerner et al, 1985; Wilson, 1985) apoyan estas formulaciones: que las respuestas de contaminación indicando trastornos en los límites ocurren pri- mordialmente en pacientes esquizofrénicos, mientras que las respuestas de confabulación indicando dificultades en la constancia evocativa, ocurren primordialmente en pacientes fronterizos.

Formas menos serias de psicopatología (por ejemplo, depresión, neurosis, etc.) ocurren en personas cuyas capacidades para la constancia de límites, de reconocimiento, objeto y self están casi intactas (Blatt, 1974, 1991, 1995; Blatt \& Shichman, 1983; Blatt \& Zuroff, 1992) pero cuyas dificultades corresponden a disrupciones particulares en la integración de esquemas del self y los otros, y en el establecimiento de un sentido de "nosotros". Debido a dificultades interpersonales marcadas, algunos de estos pacientes menos trastornados se preocupan intentando estabilizar su sentido de self, mientras que otros pacientes se preocupan intentando estabilizar su sentido de los otros. Algunos pacientes centran su atención en el intento de lograr un grado de constancia de self mientras que otros en el intento de lograr algún grado de constancia del objeto. Dos formas principales de depresión (eso es, la dependiente y la autocrítica; Beck, 1983; Blatt, 1974; Blatt, D'Afflitti \& Quinlan, 1976) y dos formas principales de desórdenes de personalidad (histeria y obsesión compulsiva; por ejemplo, Ouimette, Klein, Anderson, Riso \& Lizard, 1994) pueden diferenciarse dependiendo de si los intereses y preocupaciones del individuo se centran primordialmente en temas de autodefinición (por ejemplo, temas de poder, control, autovalor) o en temas acerca 
de la disponibilidad y confiabilidad de los otros para el apoyo emocional (Blatt, 1991, 1995; Blatt \& Shichman, 1983). Así, varios estudios de representaciones mentales en muestras de casos clínicos han proporcionado nuevas formas de entender un rango amplio de psicopatología, incluyendo la esquizofrenia (Auerbach \& Blatt, 1996, 1997; Blatt Schimek \& Brenneis, 1980; Blatt \& Wild, 1976; Blatt et al., 1975), la patología borderline (Auerbach \& Blatt, 1996; Blatt, 1990; Blatt \& Auerbach, 1988, Diamond et al., 1990; Gruen \& Blatt, 1990; Nigg, Lohr, Western, Gold \& Silk, 1992; Western, Lohr, Silk, Gold \& Kerber, 1990), la depresión (Blatt, 1974; Blatt \& Homann, 1992; Blatt \& Maroudas, 1992; Cicchetti \& Aber, 1986; Homann, 1991; Zuroff \& Fitzpatrick, 1995), y dos tipos principales de desórdenes de personalidad (Blatt, 1991, 1995; Blatt \& Shichman, 1983; Ouimette et al., 1994).

\section{LA EVALUACIÓN DE LAS}

\section{REPRESENTACIONES MENTALES}

El énfasis contemporáneo en procesos cognitivos y representación mental no sólo ha transformado la comprensión actual de la psicopatología, sino que también ha llevado a nuevas formas de pensar acerca de la evaluación de la personalidad (Blatt, 1990; Leichtmen, 1996a, 1996b) y al desarrollo de varias nuevas e importantes aproximaciones para la evaluación de las respuestas de técnicas proyectivas como el Rorschach y el test de aper- cepción temática. Muchos de los nuevos desarrollos en la evaluación de la personalidad se derivan de considerar las técnicas proyectivas como procedimientos a través de los cuales podemos observar los procesos de construcción cognitiva. Mis colegas y yo, por ejemplo, desarrollamos procedimientos para evaluar aspectos de las representaciones mentales al evaluar el contenido y la estructura de descripciones espontáneas del self y de otros significativos (Blatt, Wein, Chevron \& Quinlan, 1979; Blatt, Chevron, Quinlan, Schaffer \& Wein, 1988; Diamond, Blatt, Stayner \& Kaslow, 1991). Utilizando conceptos de teorías del desarrollo cognitivo y psicoanalíticas, hemos desarrollado un método para evaluar el grado de diferenciación y relacionamiento (Diamond, Blatt, Stayner \& Kaslow, 1991) en la descripción espontánea del self y de los otros significativos (Blatt et al., 1979, 1988).

\section{LA ESCALA DE DIFERENCIACIÓN- \\ RELACIONAMIENTO}

Nuestro grupo de investigación desarrolló la "escala de diferenciación-relacionamiento" tomando como insumos las formulaciones teóricas y observaciones clínicas acerca de los procesos muy tempranos de articulación de límites (Blatt \& Wild, 1976; Blatt, Wild \& Ritzler, 1975; Jacobson, 1964; Kernberg, 1975, 1976), de los procesos de separación-individuación (Coonerty, 1986; Mahler, Pine \& Bergman, 1975), de la formación de un sentido de self (Stern, 1985) y del desarrollo de cre- 


\section{Cuadro $\mathrm{N}^{\circ} 3$ \\ Diferenciación-relacionamiento de las representaciones del self y del otro significativo*}

\section{Nivel / Punto en escala}

1. Frontera del self-otro comprometida

2. Confusión en frontera selfotro

3. Mirroring self-otro

4. Idealización o denigración del self-otro

5. Consolidación tenue y semidiferenciada de representaciones por medio de la escisión (polarización) o por énfasis en las propiedades de partes concretas

6. Constancia (cohesión) emergente y ambivalente del self $y$ un sentido emergente de relacionamiento

7. Self y otros consolidados y constantes (estables) en relaciones unilaterales

8. Self y otros cohesivos individuados y empáticamente relacionados

9. Despliegue del self y otros recíprocamente relacionados e integrados

10. Construcciones creativas e integradas del self y el otro en relaciones empáticas y recíprocamente afinadas.

\section{Descripción}

Falta o ruptura con el sentido básico de cohesión física o integridad de las representaciones.

Self y el otro son representados como físicamente intactos y separados, pero sentimientos y pensamientos son amorfos, indiferenciados o confusos. La descripción puede consistir en una sola cualidad impresionista y global o un mar de detalles con un sentido de confusión y vaguedad.

Las características del self y del otro, como apariencia física o cualidades, forma y tamaño corporal son virtualmente idénticas.

Intento de consolidar las representaciones sobre la base de idealización del self-otro unitaria y sin modulación o denigración. Descripciones extremas y unilaterales.

Oscilación marcada entre cualidades dramáticamente opuestas o un énfasis en aspectos externos manifiestos.

Consolidación emergente de aspectos dispares del self y del otro en una integración algo indecisa, equívoca o ambivalente. Una lista de características convencionales apropiadas, pero les falta un sentido de peculiaridad. Movimiento tentativo hacia un sentido más individuado y cohesivo del self y el otro.

Los pensamientos, sentimientos, necesidades y fantasías son diferenciados y modulados. Tolerancia e integración crecientes de aspectos dispares. Distinción de cualidades y características. Comprensión simpática de otros.

Sentido del self y de otros cohesivo, matizado y relacionado. Un sentido definitivo de identidad y un interés en relaciones interpersonales y una capacidad para entender la perspectiva de otros.

Sentido cohesivo del self y de los otros en relaciones recíprocas que transforman a ambos, el self y el otro, en formas complejas y continuo despliegue.

Relaciones recíprocas integradas con una apreciación que uno contribuye a la construcción de significado en relaciones interpersonales complejas.

\footnotetext{
* Tomado de Diamond, D., Blatt, S.J., Stayner, D., \& Kaslow, N. (1991). Manual de investigación (inédito). Yale: Universidad de Yale.
} 
cientes niveles de maduración del relacionamiento interpersonal (Blatt \& Blass, 1990, 1996). La escala es de 10 puntos, en los cuales se valoran los siguientes: falta de diferenciación entre self y el otro (niveles 1 y 2); el uso de mirroring (nivel 3), idealización del self-otro o denigración (nivel 4), y la oscilación entre atributos polarizados negativos y positivos (nivel 5) como maniobras para consolidar y estabilizar representaciones; una emergente representación diferenciada, constante e integrada del self y el otro con creciente tolerancia a la ambigüedad (niveles $6 \mathrm{y}$ 7); representaciones del self y el otro como empáticamente interrelacionadas (nivel 8); representaciones del self y el otro en interacciones recíprocas y mutuamente facilitadoras (nivel 9); y representaciones integradas reflectivamente construidas del self y de los otros en relaciones recíprocas y mutuas (nivel 10). En términos generales, valores mayores de relacionamiento diferenciado en las descripciones del self y del otro están basados en la incrementada articulación y estabilización de esquemas cognitivo-afectivos y en la incrementada apreciación de relacionamientos mutuos y empáticamente afinados.

Esta escala, resumida en el cuadro 3, está basada en la asunción que el desarrollo psicológico ocurre a lo largo de dos ejes de desarrollo fundamentales: a) hacia la emergencia de un sentido de autodefinición consolidado, integrado e individuado y b) la formación de relacionamiento mutuo y empáticamente afinado con otros significativos (Aron, 1996; Benjamin, 1995; Blatt, 1991;
Blatt \& Blass, 1990, 1996; Jordan, 1986; Miller, 1984; Mitchell, 1988; Stern, 1985; Surrey, 1985). La diferenciación y el relacionamiento son dimensiones interactivas (Blatt \& Blass, 1990, 1996; Blatt \& Shichman, 1983; Sander, 1983) que se desdoblan durante el desarrollo (véase también a Kegan, 1982; Mitchell, 1988; Ogden, 1986). La interacción dialéctica entre estas dos dimensiones fundamentales del desarrollo de autodefinición y relacionamiento facilitan el surgimiento y la consolidación de niveles crecientemente maduros, ambas de la autoorganización y del relacionamiento empático y afinado intersubjetivamente (Blatt \& Shichman, 1983; Blatt \& Blass, 1990, 1996). Esta escala asume que, con el desarrollo psicológico, las representaciones del self y del otro se vuelven crecientemente diferenciados e integrados, y comienzan a reflejar una apreciación incrementada del relacionamiento mutuo.

Con respecto a la dimensión de la diferenciación, la escala refleja, en los niveles más bajos, cómo se comprometen los límites en relación con la conciencia básica del cuerpo, las emociones y los pensamientos. Niveles subsiguientes de la escala reflejan una visión unitaria y no modulada del self y el otro como extensiones de cada uno o como imágenes de espejo (eso es, en torno a un sentido exagerado de la bondad o la maldad de la figura descrita). En el próximo nivel, estos aspectos exagerados del self y el otro se alternan en una yuxtaposición de extremos polarizados (eso es, todo bueno o todo malo). 
Niveles más altos reflejan una capacidad incrementada de integrar los aspectos disimilares del self y el otro y una incrementada tolerancia a la ambivalencia y ambigüedad (Kernberg, 1977).

La escala también refleja una tendencia hacia una mutualidad empáticamente afinada en relaciones interpersonales complejas. En niveles más bajos, el sentido de relacionamiento en representaciones puede involucrar el estar controlado por el otro (por ejemplo, tratando de resistir los ataques de un otro que es considerado como malo o destructivo). En niveles crecientemente más altos, el relacionamiento puede expresarse primariamente en interacciones paralelas, en expresiones de cooperación y mutualidad, en la comprensión de la perspectiva del otro, o en expresiones de reciprocidad empáticamente afinadas (Blatt \& Blass, 1990, 1996). En los niveles más altos, las descripciones reflejan un sentido de su propia contribución a las matrices complejas y relacionales que determinan percepciones, atribuciones y construcción de significado.

Estos diez niveles de diferenciaciónrelacionamiento fueron establecidos sobre la base de hallazgos clínicos y de desarrollo y reflejan lo que generalmente se considera como distinciones clínicamente significativas en la transición de relaciones objeto gruesamente patológicas a las que son intactas y hasta saludables. Valores más altos de diferenciación-relacionamiento reflejan un grado mayor de salud psicológica. En teoría, la diferenciaciónrelacionamiento, niveles 8,9 y 10 , son indicativos de salud mental, y la diferenciación-relacionamiento nivel 7 (consolidación de constancia de objeto) es considerada como un prerrequisito para el funcionamiento psicológico e interpersonal normal.

La confiabilidad alcanzada entre los valores de distintos evaluadores y en las repruebas de este procedimiento de calificación, se encuentra en niveles aceptables (Stayner, 1994) y hallazgos de investigación apoyan la validez de esta escala como medida del nivel de funcionamiento psicológico y de cambio terapéutico (por ejemplo, Blatt, Auerbach \& Aryan, 1997; Blatt, Tayner, Auerbach \& Behrends, 1996; Diamond et al., 1990; Diamond et al., 1991).

\section{CAMBIOS EN LA REPRESENTACIÓN}

\section{MENTAL EN EL PROCESO TERAPÉUTICO}

Mis colegas y yo (Blatt, et al., 1996; Blatt, et al., 1998; Blatt et al., 1991; Diamond et al., 1990; Gruen \& Blatt, 1990) usamos la "escala diferenciaciónrelacionamiento" para evaluar cambios en las descripciones del self y de los otros significativos hechas por adolescentes y adultos jóvenes internados, severamente trastornados y con resistencias a la terapia, al principio y hacia el final de un tratamiento internado, intensivo, de largo aliento (más de un año), comprensivo, e informado psicodinámicamente. Los cambios en las descripciones del self y de los otros significativos (es decir, madre, padre y terapeuta) obtenidos al ser internados y al ser dados de alta fueron correlacionados con estimados de los cambios en el nivel 
de funcionamiento psicológico. Estos últimos fueron evaluados independientemente por medio de los informes clínicos preparados - como rutina- en los mismos tiempos (al ser internado-dado de alta) por un equipo de tratamiento interdisciplinario que incluía al terapeuta individual del paciente. Los informes de caso utilizados para derivar estos puntajes eran evaluaciones extensas y conductualmente orientadas que habían sido preparadas rutinariamente al ser internado y en intervalos de seis meses, incluyendo la terminación de la terapia, por varios miembros del personal de tratamiento. El nivel de funcionamiento psicológico de cada paciente, al ser internado y dado de alta, fueron evaluados sobre la base de estos informes de caso clínicos con la Escala de Evaluación Global (Global Assessment Scale -
GAS) (Endicott et al., 1976), una escala de 100 puntos de severidad de psicopatología. La evaluación independiente del mejoramiento clínico durante el tratamiento, fue realizada por un psicólogo clínico con experiencia que había alcanzado un nivel alto de confiabilidad interevaluador con su procedimiento de puntuación (correlación entre clase 0.87 ) en una muestra de pacientes ambulatorios crónicamente trastornados. Las descripciones del self y de figuras significativas fueron calificadas por otros evaluadores que desconocían la identidad de los pacientes y del momento en el tratamiento del cual se tomó la muestra del material relevante.

En esta investigación de cambios en la representación mental en el proceso de tratamiento, asumimos que si varias formas de psicopatología involucraban

\section{Cuadro $\mathrm{N}^{\circ} 4$ \\ Correlaciones parciales después de un año de tratamiento entre el nivel de funcionamiento clínico (GAS score) y la diferenciación-relacionamiento de representación del objeto (OR) controlando por niveles iniciales de GAS y OR1}

\begin{tabular}{llc}
\hline & $\begin{array}{c}\text { Diferenciación- } \\
\text { relacionamiento }\end{array}$ & Duración \\
\hline Madre & $.52^{* * * *}$ & .15 \\
Padre & $.31^{*}$ & .11 \\
Terapeuta & $.50^{\star * * *}$ & -.07 \\
Self & $.52^{\star * * *}$ & .15 \\
\hline
\end{tabular}

$\mathrm{N}=40$

${ }^{*}=\mathrm{p}<.05$

$* * * *=p<.0001$

1 Derivado de: Blatt, S.J.; Stayner, D.; Auerbach, J. \& Behrends, R.S. (1996). Change in object and self representations in long-term, intensive, impatient treatment of seriously disturbed adolescent and young adult, en Psychiatry: Interpersonal and biological processes 59, 82-107. 
distorsiones en la representación del objeto y del self, y que si en el desarrollo normal los apegos satisfactorios logrados en la niñez resultan en la formación de esquemas interpersonales crecientemente maduros, entonces las interacciones constructivas entre el paciente y el terapeuta deberían facilitar las revisiones de representaciones deterioradas o distorsionadas del self y el objeto y llevar al desarrollo de esquemas del self y el objeto más integrados y maduros (Behrends \& Blatt, 1985; Blatt \& Behrends, 1987; Blatt et al., 1975; Blatt, Wiseman, PrinceGibson \& Gatt, 1991). Nuestra asunción básica es que la relación terapéutica crea un proceso a través del cual esquemas interpersonales deteriorados o distorsionados son abandonados, retrabajados y transformados en representaciones cognitivo-afectivas más adaptativas del self y el otro. De ahí que, hacia el final del tratamiento, las representaciones debieran ser más diferenciadas e integradas, con indicaciones de mayor capacidad para el relacionamiento interpersonal mutuo.

Los cambios en la representación mental (eso es, en diferenciación-relacionamiento) del self y los otros significativos estuvieron correlacionados con la evaluación independiente de cambios en el nivel del funcionamiento clínico (eso es, valores en el GAS), controlando por los niveles iniciales de ambos conjuntos de variables (Tiempo 2/Tiempo 1). Como indica el cuadro 4, se encontró una relación altamente significativa entre el grado de mejora clínica después de un año de tratamiento y el incremento en la diferenciaciónrelacionamiento en las descripciones del self y de los otros significativos. Las evaluaciones independientes del grado de mejoramiento clínico utilizando los resultados GAS estuvieron correlacionados en un grado altamente significativo $(\mathrm{p}<.001)$ con incrementada diferenciación-relacionamiento en las descripciones de la madre, el terapeuta, de sí mismo, y, a menor grado significativo $(\mathrm{p}<.05)$, con la descripción del padre (Blatt et al., 1996). Así que el progreso terapéutico estuvo asociado con la incrementada diferenciación de figuras significativas, especialmente la madre y el terapeuta, y con una incrementada capacidad para representar relacionamiento interpersonal mutuo. Estos cambios en las dimensiones estructurales de las representaciones fueron independientes del cambio en la extensión o duración de la descripción. En términos generales, aquellos en nuestra muestra que estaban seriamente trastornados y con resistencia al tratamiento tenían representaciones del self y de los otros significativos al inicio del tratamiento, que estaban, en el mejor de los casos, dominadas por una representación del self y de los otros polarizada y casi siempre intensamente negativa y denigrante (nivel 4). Al ser dado de alta, los pacientes juzgados de haber tenido mayor cambio terapéutico habían logrado una consolidación de constancia de objeto (nivel diferenciación relaciomiento 7), mientras que los pacientes con menor mejoría sólo habían logrado la emergencia de constancia 
(nivel 6), eso es, una habilidad emergente para tolerar y comenzar a integrar aspectos contradictorios de figuras significativas en sus vidas. Tomado en conjunto, estos análisis (Blatt et al., 1996; Blatt et al., 1997) indican que el tratamiento de pacientes internados, de largo plazo y psicoanalíticamente orientados, resultan en incrementos sustanciales de la diferenciación-relacionamiento en las representaciones del self y de otros significativos, en el caso de pacientes seriamente trastornados y con resistencias al tratamiento.

Específicamente, las representaciones del self y de los otros significativos se trasladaron de un nivel de polarización y escisión (eso es, descripciones exageradas, unilaterales o denigrantes) a niveles de constancia objetal (eso es, descripciones involucrando una integración de elementos contradictorios y dispares). Las investigaciones futuras necesitan ser dirigidas hacia la comprensión de los procesos a través de los cuales el proceso terapéutico lleva a estos cambios en los esquemas cognitivo-afectivos (Blatt \& Behrends, 1987). Dicha investigación también debe esclarecer los procesos a través de los cuales estos cambios en las representaciones del self y los otros se relacionan con cambios en un rango amplio de procesos cognitivos y en la calidad de las relaciones interpersonales, ambos en contextos clínicos (eso es, la relación terapéutica), así como en las experiencias interpersonales en términos más generales.

Recientemente mis colegas y yo (Blatt, Diamond \& Coonerty, 1999) también hemos aplicado este modelo conceptual de niveles de diferenciación-relacionamiento de representaciones mentales a las respuestas Rorschach. Esta escala para la evaluación de respuestas Rorschach es paralela a la escala para evaluar aspectos de las descripciones del self y otros significativos. Los dos niveles inferiores son usados para identificar respuestas Rorschach que indican trastornos en límites -sea en una aprehensión global e indiferenciada de aniquilamiento (nivel 1) o en un temor más diferenciado de destrucción (nivel 2)-. El nivel 3 es utilizado para respuestas Rorschach que indican la parcial emergencia de individuación expresadas en imágenes de perfiles, mirroring o pairing. Los niveles 4 y 5 son contabilizados para respuestas que indican un intento en establecer un sentido diferenciado del self y de los otros a través del uso de conceptos extremos o polarizados. Los niveles 6 y 7 son usados para respuestas que contienen una indicación de un sentido emergente de constancia en las representaciones del self y del otro; los niveles 8 y 9 indican respuestas en las cuales individuos integrados e individuados son representados en relaciones interpersonales compartidas o recíprocas. El nivel 10 es para respuestas que indican que el individuo tiene alguna conciencia de su propia contribución a su construcción de significado en relaciones interpersonales complejas. 


\section{Cuadro $\mathrm{N}^{\circ} 5$ \\ Diferenciación-relacionamiento de respuestas Rorschach}

I. Trastorno fronterizo: frontera self-otro comprometido (pérdida de integridad y cohesión física básica)

II. Trastorno fronterizo: confusión en frontera self-otro (fronteras afectivo-intelectual están confundidos, fusionados o comprometidos)

III. Surgimiento de representaciones individuadas (un sentido de self y otro parcial o no totalmente independiente)

IV. Consolidación y estabilización de representaciones basadas en extremos (idealización o denigración)

V. Semi-diferenciación (diferenciación tentativa lograda a través de alternancia de extremos)
I. Respuestas de contaminación y temas de destrucción total (por ejemplo, "explosión de bomba atómica", "todo vuela en pedazos; todo se ha perdido", "el fin del mundo: invierno nuclear", "arrasamiento").

II. Respuestas de confabulación y temas de hundimiento o destrucción en el cual una persona o fuerza relativamente poderosa destruye a otro más pequeño, débil y pasivo. Una figura poderosa se traga o destruye un animal, una persona o una cosa usualmente pequeña o incompleta (por ejemplo, "un animal tragándose algo", "dos bebés muertos con un cuchillo traspasándolos", "dos mujeres despedazando a un bebé").

IIIA. Respuestas de perfil-sombra: ("perfil de un hombre", "contorno de una cara", "sombra de una casa", "ve su sombra a su costado").

IIIB. Mirroring: ("una niña observándose en un espejo", "mirando al reflejo en el agua").

IIIC. Pairing: ("dos mujeres exactamente iguales", "los mismos dos animales", "dos personas que se parecen mucho"; énfasis en uniformidad y similitud con respecto al otro, siendo similar al self).

IV. Temas de omnipotencia o insignificación; fortaleza o debilidad; énfasis en tamaño, sea grande o pequeño; significativo o insignificativo (por ejemplo, "el tótem" ; "magia india polarizada, inmodulada"; "poder diabólico"; "figura poderosa espeluznante"; "rey"; "gigante"; o ser "pequeño"; "casi como un gusano en comparación a otros"; "como nada, diminuto y débil").

V. Lucha entre la vida y la muerte, lucha por sobrevivir intentando hacer algo pero fracasando, peleando y siendo dañado. Luchas de empujar y jalar, luchas físicas literales involucrando ambivalencia, conflicto y confusión respecto de acercarse y alejarse, ayudar o rechazar a otros, destruir o crear una cosa, persona o animal, cambiando y alternando, mirando y corriendo en diferentes direcciones (por ejemplo, "agarrado de las manos pero sintiéndome con miedo y yendo a casa", "jugando juntos pero sintiéndome raro y largándome"). 


\section{Cuadro $N^{\circ} 5$ (continuación) \\ Diferenciación-relacionamiento de respuestas Rorschach}

VI. Surgimiento de constancia y un sentido emergente de relacionamiento

VII. Constancia de objeto consolidada y surgimiento de un sentido de relacionamiento

VIII. Self y otro cohesivo, individuado y empáticamente relacionado

IX. Relación recíprocamente relacionada integradora entre el self y el otro

X. Construcción integradora y constructiva del self y el otro al Rorschach, con alguna conciencia autorreflexiva de las implicancias de sus respuestas como revelando aspectos particulares del self.
VI. Figuras involucradas en actividades relativamente consistentes pero no especificadas que cualquier persona o animal podría hacer (eso es, corriendo, escalando, jugando, peleando, parándose, hablando, discutiendo, etc.).

VII. Sentido estable del self en relaciones unilaterales, en las cuales las acciones son únicas y congruentes con la definición del objeto de tal manera que sólo ese tipo de persona podría o debería realizar ese tipo de acción (por ejemplo, "un sacerdote dando su bendición"), pero en el cual el otro es insinuado mas no identificado o presente explícitamente. La relación es esencialmente unilateral con una persona actuando en manera diferenciada sobre una persona que es relativamente pasiva.

VIII. Sentido estable del self en el cual individuos diferenciados participan en una relación compartida. Individuos independientes colaborando en actividades o compartiendo perspectivas.

IX. Interacciones entre dos objetos diferenciados e independientes que tienen efectos recíprocos en cada uno o que implica una relación en desarrollo y despliegue.

X. Conciencia de la naturaleza relativa del proceso de respuesta y de asumir responsabilidades e implicancias de sus respuestas, como aspectos reveladores de uno mismo.

\section{ILUSTRACIÓN DE UN CASO}

Para ilustrar el valor de esta aproximación al Rorschach permítanme presentar la información de un caso clínico.

El paciente A., una mujer soltera, tenía 13 años al momento de ser internada, por tercera vez, en un hospital psiquiátrico. Los padres de A. se habían separado y se divorciaron cuando tenía tres años y medio. Ellos tenían serias dificultades maritales que comenzaron poco después del nacimiento del hermano de A., cuando ella tenía dos años. Se había informado que el padre de A. era un alcohólico y su hermano sufría lo que se describía como una "crisis nerviosa". El tío materno de A. había sido hospitalizado varias veces por trastornos emocionales no especificados.

A. fue producto de un parto normal y logró hitos de desarrollo dentro de los 
límites temporales normales. Sus dificultades actuales surgieron del trasfondo de amargura crónica entre sus padres divorciados y de sus antiguas perturbaciones interpersonales y de personalidad. Al momento de la separación y divorcio de sus padres, cuando A. tenía tres años y medio, ella comenzó a tener problemas de alimentación. También tenía pataletas frecuentes y dificultades al separarse de su madre, problemas que persistieron hasta sus cinco años. Cuando tenía cuatro o cinco A. fue sometida a evaluación psiquiátrica debido a estas dificultades. Aproximadamente a los ocho comenzó a creer que su cuerpo estaba poseído por el demonio y a tener alucinaciones de la voz y la cara del diablo. A los diez años tenía frecuentes discusiones con su madre y padre, y rehusaba ir a la escuela. Su desempeño escolar se deterioró, su relación con la madre se volvió incrementadamente hostil, turbulenta y violenta, y comenzó a experimentar con cannabis y alcohol. Ella eventualmente progresó a diazepam (Valium), metaqualone (Quaalude) y heroína intravenosa. Aunque sólo tuvo contacto mínimo con su padre durante los nueve años después que sus padres se separaran y divorciaran, ella se mudó al hogar de éste a los doce años porque su madre ya no podía manejarla.

A. fue internada brevemente por primera vez cuando tenía doce años después de un intento de suicidio con una sobredosis de diazepam. Fue dada de alta después de unos días y continuó su deterioro durante el año siguiente. Su abuso de drogas empeoró, al igual que sus alucinaciones visuales y auditivas. Ella se mantuvo crónicamente deprimi$\mathrm{da}$, ansiosa, suicida y afectivamente lábil, con períodos breves y ocasionales de elación y euforia, pero se negó a recibir psicoterapia ambulatoria. Fue hospitalizada brevemente una vez más antes de ser internada en nuestra pequeña clínica privada y de tratamiento de largo plazo. Para el tiempo de esta tercera hospitalización psiquiátrica, había sido diagnosticada como víctima de una severa depresión psicótica con tendencias paranoicas marcadas en una personalidad borderline con rasgos mixtos histriónicos y compulsivos.

En una evaluación clínica al momento de internamiento, A. fue descrita como deprimida en apariencia y conducta. Reservada, con una dieta e higiene pobres, y distraída con frecuencia por estímulos internos, ella era incapaz de sostener una conversación y estaba insegura de lo que ocurría en su entorno. Su equipo de tratamiento describió que actuaba su depresión en vez de comunicarla verbalmente, e informó que era obsesiva, preocupada y rumiaba, con un nivel psicótico de ambivalencia. Sus mayores defensas contra la depresión eran la intelectualización y el aislamiento del afecto.

Durante el curso de la hospitalización de A., su puntaje GAS aumentó de 22 (incapaz de funcionar en casi todas las áreas) al ser internada, a 43 (síntomas y deterioro serios) al ser dada de alta. Revisiones periódicas del tratamiento de A., durante los 19 meses de hospitalización, reportaron que su autoestima había mejorado notablemente; se había 
vuelto menos vulnerable a la descompensación psicótica y era más capaz de utilizar las relaciones con otros para superar las crisis. También era capaz de darse cuenta de que su involucramiento con drogas era un sustituto por la atención que ella sentía que no podía obtener de otras maneras. Aunque todavía era vulnerable a regresiones psicóticas, fue dada de alta del hospital y pasó a un programa residencial porque sus padres no podían continuar con el pago del tratamiento intensivo. Vamos ahora a considerar las descripciones de A. del self y de los otros significativos, al igual que sus respuestas Rorschach al momento de su internamiento y al ser dada de alta.

\section{Cuadro $\mathrm{N}^{\circ} 6$ \\ Internamiento}

Paciente A: Representaciones del self y objeto

MADRE: (D-R=4) Preocupada, agresiva, triste y solitaria.

Pregunta: (¿Algo más?) No.

PADRE: $(D-R=4)$

SELF: $(D-R=5)$

Extrovertido, generoso, considerado, comprensivo. Eso es todo.

Depende de cómo me siento. Algunas veces soy extrovertida pero otras veces soy reservada. No sé.

Pregunta: (¿Qué otra cosa?) Yo no quiero describirme a mí misma (¿Por qué?) Porque me molesto cuando lo hago. (¿Me puedes decir qué es lo que te molesta?) Soy o muy arrogante o demasiado modesta para contestar algo así.

TERAPEUTA: ( $D-R=6) \quad$ Dulce, que apoya, confiable y que se preocupa.

$1 \frac{1}{2}$ Años (dada de alta)

MADRE: ( $D-R=6) \quad$ Ella es dulce, se preocupa, terca. Ella intenta mucho, trabaja duro. Algunas veces es dominante. Mide como 5 pies con 5 pulgadas, pesa unas 120 libras, pelo rizado, no tiene mucho arriba, algo de pecho plano. Es terca.

Pregunta: (¿Dominante?) Algunas veces es demasiado para mí - todas estas cualidades son demasiado para mí. A ella no le gusta ceder.

PADRE: (D-R=7) Gracioso, preocupado por mí, bondadoso. Es generoso conmigo. Trata de ser comprensivo. No puede mantener muy bien un secreto. Como 5 pies con 9 pulgadas, 185 libras. Un poco... regordete. Perdiendo pelo. Una persona bien intencionada. Felizmente casado en el presente.

Pregunta: (¿Felizmente casado en el presente?) Realmente casado felizmente. El tiene una boca grande. Eso es todo.

SELF: (D-R=7)

Solitaria, insegura. Escondiéndome detrás de una fachada. Tiene sentido común, opiniones anormales. Una de mis opiniones anormales es que las personas que quieren matarse a sí mismas deben ser permitidas a matarse a sí mismas; y no me estaba refiriendo a mí misma. Madura -puede ser madurarealmente no lo he actuado durante las pruebas psicológicas. Yo más o menos me hice la tonta. Debí tener más seguridad y confianza.

TERAPEUTA: ( $D-R=8) \quad$ Estoy tratando de pensar en una palabra. Tiene tacto al abordar temas. Esa no era la palabra en la estaba pensando, no es brusca, puede decir las cosas de una mejor forma. Ella puede decir las cosas en una forma mejor que no suena tan intimidante o tan cruel. Ella es dulce, generosa y tiene una moralidad alta. Ella es una buena persona. Tiene estándares altos. 
Al ser internada, A. describía a su madre en términos negativos y disfóricos. En un contraste marcado, caracterizaba a su padre como teniendo cualidades positivas. Debido a que estos rasgos también eran relacionales en tono, ellos sugerían que, por lo menos en un nivel manifiesto, A. visualizaba su relación con su padre como considerablemente más protectora que con la madre. Aunque sus representaciones de la madre y el padre estaban escindidas en términos de todo malo y todo bueno, esta polarización también indicaba una diferenciación y organización básica en sus procesos de pensamiento.

La autodescripción de A. durante su admisión, aunque básicamente negativa en tono, contenía polaridades perplejas que sugerían un reconocimiento inicial de los aspectos contradictorios de sí misma y una realización de que su autoconcepto era altamente dependiente de su estado afectivo. Aunque su sensibilidad a sentimientos de exponerse, vergüenza y grandiosidad, sugerían un trastorno narcisista, al igual que era consistente con su orientación autocrítica y paranoica; su reconocimiento de algunos de los antecedentes de su autovariabilidad sugerían que ella podría ser capaz de desarrollar diferenciaciones más sutiles. En contraste con su descripción polarizada de sus padres, su autodescripción involucraba un nivel más alto de diferenciación. Ambas imágenes positivas y negativas estaban presentes, al igual que su reconocimiento, que necesitaba integrar ambas series de cualidades para poder comprenderse a sí misma. Su tendencia hacia la introspección y su disposición a la autodescripción, a pesar de sus reservas acerca de la tarea, sugerían un potencial a que se comprometiera constructivamente en una psicoterapia. Pero generalmente su autodescripción era fragmentada y sin integración. Estaba organizada alrededor de polaridades rudimentarias de atributos que estaban directa o indirectamente expuestas en las descripciones de sus padres.

Al ser internada, A. caracterizó a su terapeuta como a su padre, en términos positivos e idealizados que enfatizaban el potencial para un relacionamiento interpersonal. Las notas de la revisión de la terapia preparada por su terapeuta tres meses después de su hospitalización, describían a A. como muy necesitada y como desesperadamente esperanzada en que su terapeuta femenina se convirtiera en la madre deseada e idealizada que podría cuidarla y nutrir1a. A. reaccionó con sentimientos de rechazo y cólera a la reprogramación de sus horas de terapia y a la ausencia de su terapeuta durante unas vacaciones. Ella se volvió psicótica por momentos en respuesta a estas pérdidas percibidas.

Al momento de terminar la terapia, se notó un cambio significativo, como reflejo de una considerable mejora clínica en las descripciones obtenidas al momento de que A. fuera dada de alta del hospital. El foco de la descripción de su madre cambió de disfórica a aspectos relativamente no conflictivos de su relación. Ella describía a su madre en términos físicos $\mathrm{y}$, aunque todavía consideraba a su madre como emo- 
cionalmente insuficiente y limitada en crianza, por primera vez A. utilizó una mezcla más modulada de términos positivos y negativos que retrataban a su madre como capaz de proveer para ella. A los 18 meses A. estaba comenzando a integrar rasgos físicos más objetivos de su madre, con una comprensión más equilibrada, calificada y diferenciada de la personalidad de su madre.

Un cambio constructivo similar ocurrió en la descripción más integrada de su padre. Ella ahora lo caracterizaba en términos positivos pero no idealizados, que también expresaba un sentido incrementado de relacionamiento. Al igual que la madre, estos descriptores fueron moderados con calificativos y, por ende, parecían menos polarizados y absolutos. Ella reconocía que su padre tenía ambas limitaciones y fortalezas. La descripción de su padre, al igual que la de su madre, también se hizo más objetiva y realista con la introducción de sus características físicas, aunque estos términos (por ejemplo, "regordete") sugerían sus propias necesidades. El uso de la palabra "preocupados" para describir a ambos (padre y madre) en ese momento, indicaba un mayor sentido de relacionamiento con ellos.

Los temas depresivos continuaban en la descripción de A. al momento de ser dada de alta, pero eran menos dominantes e intensos. Su preocupación con el suicidio también indicaba su disforia continua, aunque ahora ella era capaz de modular esta preocupación a través de la intelectualización. El cambio principal al ser dada de alta, sin embar- go, era su incrementada capacidad de autorreflexión. A. finalmente, había comenzado a pensar sobre ella misma en un modo operacional formal.

En contraste con su negativa de describir a su terapeuta en un año, A. ahora la describía en términos positivos. Ella enfatizó la calidad de la relación con su terapeuta -específicamente que el compartir sentimientos, pensamientos y experiencias podían enriquecer experiencias personales-. Es notable que la descripción de A. de su terapeuta al ser dada de alta, enfatizaba la capacidad en la terapeuta que A. también claramente valoraba en sí misma -a saber, la habilidad de seleccionar las palabras o frases apropiadas para comunicar efectivamente sus pensamientos, sentimientos $\mathrm{y}$, especialmente, para moderar sentimientos y deseos destructivos-. Similarmente, el reconocimiento de A. de los altos estándares morales de su terapeuta asemejaba su propia formación de un pensamiento operacional formal expresado en sus creencias éticas, específicamente en torno al suicidio.

Las notas de la revisión del tratamiento al momento de alta indicaban que, desde el final del primer año de tratamiento hasta el momento en que la paciente salió, siete meses después, A. se volvió crecientemente capaz no sólo de reconocer que los sentimientos hostiles que ella tenía hacia su terapeuta eran derivados de un sentido de privación y rechazo de larga data vivida con relación a su madre, sino que ella también era capaz de verbalizar estos sentimientos de privación y cólera. Reconociendo que su visión de la terapeuta 
había sido distorsionada durante buena parte del tratamiento, A. ahora era capaz de expresar sentimientos fuertes y positivos hacia su terapeuta al término del tratamiento. Las representaciones de A. del self y de los otros significativos al ser dada de alta, por ende, reflejaban un sentido más claro de diferenciación e integración, al igual que una capacidad incrementada de autoreflexión y relacionamiento interpersonal. Aunque su expresión de afecto se había convertido crecientemente moderado, equilibrado y calificado, seguía siendo disfórica.

Las crecientes expresiones de depresión de A. parecían ser paralelas a los múltiples indicadores independientes de su mejoría. El aumento en su verbalización de la depresión, en su autodescripción, aun cuando este afecto era defendido por la intelectualización, coincidía con los informes contenidos en las revisiones del tratamiento que indicaban que se cuidaba mejor a sí misma, que a veces asistía a la escuela, que estaba más conectada con los otros y que parecía menos psicótica. El paralelo entre el incremento en la disforia y un funcionamiento psicológico mejor organizado, sugiere que una mayor capacidad para experimentar y expresar sentimientos autocríticos -en vez de proyectarlos- fue una faceta importante en su mejoramiento clínico al ir pasando de una organización paranoica a una depresiva (Blatt \& Bers, 1993).

En el transcurso de su tratamiento, las autodescripciones de A. también enfatizaron crecientemente el relacionamiento interpersonal. Ella mostraba esta cre- ciente inversión en las dimensiones interpersonales a través de su reconocimiento de la presencia del examinador, sus expresiones de soledad y en el reconocimiento de su consideración hacia otros. En términos globales, por lo tanto, las descripciones de A. eran más integradas y tenían un mayor sentido de sí misma y de los otros como reales, sustanciales e interrelacionados.

Las respuestas Rorschach de A. esencialmente asemejaban los cambios notados en su descripción del self y de los otros significativos. Las respuestas que reflejaban trastornos fronterizos severos (nivel 2) que estaban presentes al momento de ser admitida fueron reemplazadas al terminar su terapia con un número de respuestas que indicaban el surgimiento de constancia objetual. Por ejemplo, al ser internada, en la lámina II, la paciente A. respondió:

- "Un demonio matando a dos personas" (la figura "matando", porque parecía que había sangre).

- "Una ardilla voladora muerta" (la cabeza y el cuerpo parecían desplegados como una ardilla voladora pero tenía sangre en ella, así que parecía muerta).

Y en la lámina III, al ser internada, respondió:

- Dos mujeres despedazando a un bebé (la forma que tenía la figura y tenían una masa en sus manos y la sangre salpicando).

- Un monstruo que está matando a gente (la forma en que los brazos estaban levantados, la figura y sangre salpicando por todas partes). 
En contraste, después de 18 meses de tratamiento, la paciente A. respondió a la lámina II en la siguiente forma:

- Dos figuras negras encapuchadas adorando el rojo, y hay pies. Están parados en un charco de sangre. (sólo la forma en que las figuras estaban configuradas. Ellas tenían brazos, piernas y pies. Ambas tenían las manos levantadas -por eso el rojo-. Estoy tratando de enojarte pero parece que no está funcionando. [¿rojo?] Porque tenían rojo en sus pies).

Y A. dio la siguiente respuesta a la lámina III al terminar su terapia:

- Dos damas africanas, trabajando sobre una olla al frente de un dios volcán. (porque eran oscuras, muy oscuras. Negras y tenían el pelo muy corto -ellas se parecían a gente que vi en National Geographic-. El dios volcán se parece a algún tipo de dios, yo pensé que sería creativa, porque los ojos y la nariz eran mucho más grandes).

Así, en contraste con los trastornos de límites expresados como amenazas a la integridad física (nivel 2) indicadas en las respuestas violentas dada al Rorschach al momento del internamiento, las respuestas al terminar el tratamiento estaban en un nivel de desarrollo superior, indicando la emergencia de constancia objetual (nivel 7) -respuestas de figuras algo peculiares dedicadas a una actividad parcialmente colaborativa-. A pesar de la presencia de contenidos atípicos en estas respuestas, estructuralmente indican un incremento en la estabilización de la representación del objeto. Es interesante notar que las respuestas al ser dada de alta, contienen elementos del nivel 10 , tal como se indica en las observaciones autoreflexivas de la paciente A. acerca de su motivación al brindar aspectos de estas respuestas -tratando de enojar al examinador y tratando de ser creativa-.

\section{RESUMEN}

Mientras nos encontramos en el umbral del siglo XXI y tenemos casi un siglo completo de experiencia en métodos proyectivos de evaluación de la personalidad, resulta importante evaluar las contribuciones realizadas con métodos proyectivos y tratar de anticipar nuevas direcciones y oportunidades. Es un tributo a Hermann Rorschach que su procedimiento para observar cómo individuos enfrentan la ambigüedad, es aún más relevante para la teoría psicológica contemporánea que cuando primero introdujo el método de manchas de tinta hace casi 80 años. Tal como ha sido discutido en este trabajo y otros previos (Blatt, 1986, 1990), el Rorschach tiene un potencial considerable para contribuir al estudio de procesos cognitivos -especialmente el mundo representacional y de cómo los individuos construyen significado-. La representación del self y del otro es actualmente uno de los principales temas en la investigación del apego y de la cognición social, y es muy relevante para la psicología y la psiquiatría clínica porque provee una 
forma de comprender la psicopatología que va más allá del simple listado de síntomas manifiestos tan predominante en la psiquiatría descriptiva actual. Un modelo del desarrollo del mundo representacional nos permite comenzar a identificar y comprender la estructura de pensamiento subyacente en varias formas de psicopatología, desde la esquizofrenia hasta las neurosis. Adicionalmente, los cambios en la estructura y el contenido de las representaciones mentales proveen una forma de evaluar los cambios importantes que ocurren en el proceso terapéutico. Y el Rorschach puede jugar un papel central en estos nuevos y excitantes desarrollos porque su ambiguo estímulo es una forma particularmente efectiva de evaluar de manera sistemática estas estructuras representacionales.

Por lo tanto, los recientes y principales desarrollos en la ciencia psicológica nos proveen la oportunidad para establecer vínculos productivos entre la teoría psicológica básica y nuestros esfuerzos clínicos. El nuevo énfasis en la representación mental debe permitirnos extender nuestro conocimiento de la psicopatología y descubrir nuevas formas para evaluar sistemáticamente en un rango amplio de trastornos psicológicos. Estos desarrollos recientes de la ciencia psicológica, no sólo pueden enriquecer nuestra práctica clínica, sino que los métodos proyectivos pueden, a su vez, proveer una metodología para enriquecer a la investigación psicológica básica y a la teoría de los procesos cognitivos. El descubrimiento de Rorschach del valor del estu- dio de cómo los individuos intentan hacer frente a estímulos ambiguos proveen un método experimental que va más allá del autoinforme y lista de chequeo -un método que permite que los investigadores puedan tener una visión más completa y comprensiva de los procesos mentales complejos y estudiarlos sistemáticamente-- Los procesos mentales provocados por el Rorschach son de vital importancia si queremos comprender en forma más completa el desarrollo psicológico normal, las disrupciones que pueden ocurrir en estos procesos de desarrollo normales, y el complejo proceso interpersonal que denominamos psicoterapia, en el cual algunos individuos son asistidos en la búsqueda de formas de hacer frente eficazmente con las disrupciones en el desarrollo. De muchas maneras, estamos en el umbral de una nueva y excitante área en la evaluación de la personalidad si tenemos el coraje de pensar en formas nuevas y creativas acerca de los vínculos entre nuestra actividad clínica y los recientes y principales avances en la ciencia psicológica básica, particularmente en los avances en nuestra apreciación de cómo los individuos desarrollan la comprensión de sí mismos y de sus relaciones interpersonales significativas. 


\section{REFERENCIAS}

Adler, G. \& Buie, D.H., Jr. (1979). Aloneness and borderline psychopathology: The possible relevance of child development and issues. International Journal of Psycho-Analysis, 60, 83-96.

Ainsworth, M.D.S. (1982). Attachment: Retrospect and prospect, en C.M. Parkes \& J. Stevenson-Hinde (Eds.). The place of attachment in human behavior (pp. 3-30). New York: Basic Books.

Aron, L. (1996). A meetings of minds: Mutuality in psychoanalysis. Hillsdale, N.J.: The Analytic Press.

Auerbach, J. S. \& Blatt, S.J. (1996). Selfrepresentation in severe psychopathology: The role of reflexive self-awareness. Psychoanalytic Psychology, 13, 297-341.

Auerbach, J.S. \& Blatt, S.J. (1997). Impairment of self-representation in schizophrenia: The roles of boundary articulation and self-reflexivity. The Bulletin of the Menninger Clinic, 61, 297-316.

Beck, A.T. (1983). Cognitive therapy of depression: New perspectives, en P.J. Clayton \& J.E. Barrett (Eds.). Treatment of depression: Old controversies and new approaches (pp. 265290). New York: Raven.

Beebe, B. (1986). Mother-infant mutual influence and precursors of self and object representations, en: J. Masling (Ed.). Empirical studies of psychoanalytic theories. Vol. 2. (pp. 27-48). Hillsdale, N.J.: Analytic Press.

Beebe, B. \& Lachmann, F.M. (1988). The contribution of the mother-infant mutual influence to the origins of self- and object representations. Psychoanalytic Psychology, 5, 305338.

Behrends, R.S. \& Blatt, S.J. (1985). Internalization and psychological development throughout the life cycle. Psychoanalytic Study of the Child, 40, 11-39. Translated and reprinted in Arbeitshefte Kinderanalyse.

Benjamin, J. (1995). Like subjects love objects: Essays on recognition and sexual differences. New Haven: Yale University Press.

Beres, D. \& Jospeh, E. (1970). The concept of mental representation in psychoanalysis. International Journal of Psycho-Analysis, 51, 1-9.

Blatt, S.J. (1974). Levels of object representation in analitic and introjective depression. Psychoanalytic Study of the Child, 29, 107-157.

Blatt, S.J. (1986). Where have we been and where are we going?: Reflections on fifty years of personality assessment. Invited contribution to Fiftieth Anniversary Edition of The Journal of Personality Assessment, 50, 343-346.

Blatt, S.J. (1990). Interpersonal relatedness and self-definition: Two personality configurations and their implications for psychopathology and psychotherapy, en J.L. Singer (Ed.). Repression and dissociation: Implications for personality theory, psychopathology \& health (pp. 299-335). Chicago: University of Chicago Press.

Blatt, S.J. (1991). A cognitive morphology of psychopathology. Journal of Nervous and Mental Disease, 179, 449458. 
Blatt, S.J. (1995). Representational structures in psychopathology, en D. Cicchetti \& S. Toth (Eds.). Rochester Symposium on Developmental Psychopathology: Emotion, cognition and representation. Vol. 6. (pp. 1-33). Rochester, N.Y.: University of Rochester Press.

Blatt, S.J. \& Auerbach, J.S. (1988). Differential cognitive disturbances in three types of "borderline" patients. Journal of Personality Disorders, 2, 198-211.

Blatt, S.J.; Auerbach, J.S. \& Aryan, M. (1998). Representational structures and the therapeutic process, en J. Masling $\&$ R. Bornstein (Eds.). Empirical investigations of events within the analytic hour (1) (pp. 63-107). Washington, D.C.: APA Books.

Blatt, S.J.; Auerbach, J.S. \& Levy, K.N. (1997). Mental representations in personality development, psychopathology, and the therapeutic process. Review of General Psychology, 1, 351374.

Blatt, S.J. \& Behrends, R.S. (1987). Internalization, separation-individuation, and the nature of therapeutic action. International Journal of Psychoanalysis, 68, 279-297.

Blatt, S.J. \& Bers, S.A. (1993). Commentary on a cognitive perspective on self-representation in depression by Z.V. Segal \& J.C. Muran, en Z.V. Segal \& S.J. Blatt (Eds.). Self representation and emotional disorders: Cognitive and psychodynamic perspectives (pp. 164170). New York: Guilford Press.

Blatt, S.J. \& Blass, R.B. (1990). Attachment and separateness: A dialectic model of the products and processes of psychological development.
Psychoanalytic Study of the Child, 45, 107-127.

Blatt, S.J. \& Blass, R. (1996). Relatedness and self definition: A dialectic model of personality development, en G.G. Noam \& K.W. Fischer (Eds.). Development and vulnerabilities in close relationships (pp. 309-338). Hillsdale, N.J.: Lawrence Erlbaum Associates.

Blatt, S.J.; Chevron, E.S.; Quinlan, D.M.; Schaffer, C.E. \& Wein, S.J. (1988). The assessment of qualitative and structural dimensions of object representations (revised edition). Unpublished research manual. Yale University.

Blatt, S.J.; D’Afflitti, J.P. \& Quinlan, D.M. (1976). Experiences of depression in normal young adults. Journal of Abnormal Psychology, 85, 383-389.

Blatt, S.J.; Diamond, D. \& Coonerty, S. (1999). Differentiation-relatedness of Rorschach responses. Unpublished research manual. Yale University.

Blatt, S.J. \& Homann, E. (1992). Parentchild interaction in the etiology of dependent and self-critical depression. Clinical Psychology Review, 12, 47-91.

Blatt, S.J. \& Lerner, H.D. (1983a). The psychological assessment of object representation. Journal of Personality Assessment, 47, 7-28.

Blatt, S.J. \& Lerner, H.D. (1983b). Investigations in the psychoanalytic theory of object relations and object representation, en J. Masling (Ed.). Empirical Studies of Psychoanalytic Theories. Hillsdale, N.J.: Erlbaum Associates.

Blatt, S.J. \& Maroudas, C. (1992). Convergence of psychoanalytic and cognitive behavioral theories of depression, 
Psychoanalytic Psychology, 9, 157190.

Blatt, S.J. \& Ritzler, B.A. (1974). Thought disorder and boundary disturbances in psychosis. Journal of Consulting and Clinical Psychology, 42, 370-381.

Blatt, S.J.; Schimek, J. \& Brenneis, C.B. (1980). The nature of the psychotic experience and its implications for the therapeutic process, en J. Strauss, M. Bowers, T.W. Downey, S. Fleck, S. Jackson \& I. Levine (Eds.). The sychotherapy of Schizophrenia (pp. 101-114). New York: Plenum.

Blatt, S.J. \& Shichman, S. (1983). Two primary configurations of psychopathology. Psychoanalysis and Contemporary Thought, 6, 187-254.

Blatt, S.J.; Stayner, D.; Auerbach, J.S. \& Behrends, R.S. (1996). Change in object and self-representations in longterm, intensive, inpatient treatment of seriously disturbed adolescents and young adults. Psychiatry: Interpersonal and Biological Processes, 59, 82-107.

Blatt, S.J., Wein, S.J., Chevron, E.S. \& Quinlan, D.M. (1979). Parental representations and depression in normal young adults. Journal of Abnormal Psychology, 88, 388-397.

Blatt, S.J.; Wiseman, H.; Prince-Gibson, E. \& Gatt, H. (1991). Object representation and change in clinical functioning. Psychotherapy, 28, 273-283.

Blatt, S.J. \& Wild, C.M. (1976). Schizophrenia: A developmental analysis. New York: Academic Press.

Blatt, S.J.; Wild, C.M. \& Ritzler, B.A. (1975). Disturbances in object representation in schizophrenia. Psychoanalysis and Contemporary Science, 4, 235-288.
Blatt, S.J. \& Zuroff, D.C. (1992). Interpersonal relatedness and self-definition: Two prototypes for depression. Clinical Psychology Review, 12, 527562.

Blum, G.S. (1961). A model of the mind. New York: Wiley.

Bowlby, J. (1969). Attachment and loss. Vol. 1 New York: Basic Books.

Bowlby, J. (1988a). Developmental psychology comes of age. American Journal of Psychiatry, 145, 1-10.

Bowlby, J. (1988b). A secure base: Clinical applications of attachment theory. London: Routledge \& Kegan Paul.

Bretherton, I. (1985). Attachment theory: Retrospect and prospect. Monographs of the Society for Research in Child Development, 50, (1 \& 2, Serial No. 209): 3-35.

Cicchetti, D. \& Aber, L.J. (1986). Early precursors of later depression: An organizational perspective, en L. Lipsett (Ed.). Advances in infant research. Vol. 3. Norwood, N.J.: Abley.

Coonerty, S. (1986). An exploration of separation-individuation themes in borderline personality disorder. Journal of Personality Assessment, 50, 501-511.

Diamond, D.; Blatt, S.J.; Stayner, D. \& Kaslow, N. (1991). Self-other differentiation of object representations. Unpublished research manual. Yale University.

Diamond, D.; Kaslow, N.; Coonerty, S. \& Blatt, S.J. (1990). Change in separation individuation and intersubjectivity in long-term treatment. Psychoanalytic Psychology, 2, 363-397. 
Endicott, J.; Spitzer, R.L.; Fleiss, J.L. \& Cohen, J. (1976). The Global Assessment Scale: A procedure for measuring overall severity of psychiatric disturbance. Archives of General Psychiatry, 33, 766-771.

Gardner, H. (1985). The mind's new science: A history of the cognitive revolution. New York: Basic Books.

Gruen, R. \& Blatt, S.J. (1990). Change in self and object representation during long-term dynamically oriented treatment. Psychoanalytic Psychology, 7, 399-422.

Homann, E. (1991). Parent- child interaction in dependent and self critical depression. Unpublished master's thesis. University of Michigan, Ann Arbor.

Jacobson, E. (1964). The Self and the Object World. New York: International Universities Press.

Horowitz, M.J. (1972). Modes of representation of thought. Journal of the American Psychoanalytical Association, 20, 793-819.

Jacobson, E. (1964). The Self and the Object World. New York: International Universities Press.

Jordan, J.V. (1986). The meaning of mutuality. Work in progress, 23, Wellesley, M.A.: Wellesley College, Stone Center.

Kegan, R. (1982). The Evolving Self: Problem and process in human development. Cambridge, M.A.: Harvard University Press.

Kernberg, O.F. (1975). Borderline conditions and pathological narcissism. New York: Jason Aronson.

Kernberg, O.F. (1976). Object relations theory and clinical Psychoanalysis. New York: Aronson.
Kernberg, O.F. (1977). Boundaries and structure in love relations. Journal of the American Psychoanalytic Association, 25, 81-114.

Leichtman, M. (1996a). The Rorschach: A developmental perspective. Hillsdale, N.J.: Analytic Press.

Leichtman, M. (1996b). The nature of the Rorschach task. Journal of Personality Assessment, 67, 478-493.

Lerner, H.D.; Sugarman, A. \& Barbour, C. (1985). Patterns of ego boundary disturbance in neurotic, borderline, and schizophrenic patients. Psychoanalytic Psychology, 2, 47-66.

Levy, K.N.; Blatt, S.J. \& Shaver, P. (1998). Attachment styles and parental representations. Journal of Personality \& Social Psychology, 74, 407-419.

Mahler, M.S.; Pine, F. \& Bergman, A. (1975). The psychological birth of the human infant. New York: Basic Books.

Main, M.; Kaplan, N. \& Cassidy, J. (1985). Security in infancy, childhood and adulthood: A move to the level of representation, en I. Bretherton \& E. Waters (Eds.). Growing points in attachment theory and research Monographs of the Society for Research in Child Development, 50, (1 \& 2, Serial $\mathrm{N}^{\mathrm{o}}$ 209): 66-104.

Miller, J.B. (1984). Toward a new psychology of women. Boston, M.A.: Beacon Press.

Mitchell, S.A. (1988). Relational concepts in psychoanalysis: An integration. Cambridge: Harvard University Press.

Nigg, J.T.; Lohr, N.E.; Westen, D.; Gold, L.J.\& Silk, K.R. (1992). Malevolent object representation in borderline personality disorder and major depression. 
Journal of Abnormal Psychology, 101, 61-67.

Ogden, T.H. (1986). Matrix of the mind: Object relations \& the psychoanalytic dialogue. Northvale, N.J.: Jason Aronson.

Osgood, C.E. (1953). Method and theory in experimental psychology. New York: Oxford.

Ouimette, P.C.; Klein, D.N.; Anderson, R.; Riso, L.P. \& Lizardi, H. (1994). Relationship of sociotropy/autonomy and dependency/self-criticism to DSMIII-R personality disorders. Journal of Abnormal Psychology, 103, 743-749.

Rorschach, H. (1921/1951). Psychodiagnostics. Bern: Huber.

Sander, L.W. (1983). Polarity, paradox, and the organizing process in development, en J. Call, E. Galenson \& R. Tyson (Eds.). Frontiers of infant psychiatry, (pp. 315-327). New York: Basic Books.

Sandler, J. \& Rosenblatt, B. (1962). The concept of the representational world. Psychoanalytic Study of the Child, 17, 128-145.

Stayner, D. (1994). The relationship between clinical functioning and changes in self and object representations in the treatment of severely impaired inpatients. Unpublished doctoral dissertation, Teachers College, Columbia University, New York.

Stern, D.N. (1985). The interpersonal world of the infant: A view from psychoanalysis and developmental psychology. New York: Basic.

Surrey, J.L. (1985). Self-in-relation: A theory of women's development. Unpublished manuscript. Stone Center for
Developmental Services and Studies, Wellesley College, Wellesley, M.A.

Westen, D.; Lohr, N.E.; Silk, K.; Gold, L. \& Kerber, K. (1990a). Object relations and social cognition in borderlines, major depressives and normals: A thematic apperception test analysis. Psychological Assessment: A Journal of Consulting and Clinical Psychology, 2, 355-364.

Wilson, A. (1985). Boundary disturbances in borderline and psychotic states. Journal of Personality Assessment, 49, 346-355.

Zuroff, D.C. \& Fitzpatrick, D.A. (1995). Depressive personality styles: Implications for adult attachment. Personality and Individual Differences, 18, 253-265. 